\title{
Study of flowering patterns of Campanula L. species using computer modeling
}

\author{
Tatyana I. Fomina ${ }^{*}$, and Eduard S. Fomin ${ }^{2}$ \\ ${ }^{1}$ Central Siberian Botanical Garden SB RAS, 630090, Novosibirsk, Russia \\ ${ }^{2}$ Institute of Cytology and Genetics SB RAS, 630090, Novosibirsk
}

\begin{abstract}
Long-term data on flowering phenology of 9 Campanula $\mathrm{L}$. species were processed using computer modeling. Modified model is proposed to describe flowering of monocarpic shoots with multi-flowered inflorescences taking into account structural inflorescence features and morphogenesis of floral zone. The flowering patterns are determined by degree of inflorescence branching and sequence of flowers opening in them. It is shown that flowering curve for simple inflorescences is unimodal: it has single peak associated with opening of terminal flower and flowers on the 1st order axes. For compound inflorescences two maxima are noted (the second of them is due to opening of flowers on the 2 nd order axes), or curve has wavy character. Regression curves describing sequence of flowers opening on the 1st order axes are different. They are $\mathrm{C}$-shaped if flowering begins in lower third of the inflorescence and then spreads divergently. More often terminal flower opens first, and is accompanied by several flowers in lower third of inflorescence, while next flowers open in divergent way. In this case the regression curves are Sshaped. The proposed model reproduces picture of individual flowering with natural variability, and can be used to describe flowering of various taxa of herbaceous plants with multi-flowered inflorescences.
\end{abstract}

\section{Introduction}

The reproductive success of plants in their seasonal development depends on the synchronization of flowering with environmental conditions that favor fertilization and seed formation. For plants with multi-flowered inflorescences, the location of flowers on the shoot and the sequence of their opening is important $[1,2]$. The dynamics of flowering can be described at different levels and by different methods, including flowering curves, the parameters of which are determined phylogenetically [3, 4].

The classical study by Primack [5] mentions that researchers give insufficient attention to the modeling of individual flowering. The phenology of flowering is determined by

${ }^{*}$ Corresponding author: fomina-ti@yandex.ru 
physiological factors, but environmental conditions affect the timing and duration of flowering, thus demanding study at the organismal level $[6,7]$.

Interest in modeling plant development at the individual level is also associated with the recent advance of automated phenotyping systems based on pattern recognition $[8,9]$. Realistic dynamic 3D models for species with multi-flowered inflorescences can only be obtained by simulating the structure of the floral zone of the shoot and its morphogenesis. Previously, we [10] attempted to construct a structural-dynamic model of flowering in several species of the Campanulaceae family. It is based on the shoot metamerism, and the presence of stable relationships between the location of flowers in the inflorescence and their flowering time.

In this paper, we propose an adaptive computer model in which all structural and dynamic dependences are extracted from the data of phenological observations. When the real data is changed, it is automatically corrected. In addition, the software implementation of the model allows to make a forecast and visualization of the flowering process with an arbitrary number of flowers (simulated shoots).

\section{Materials and methods}

The objects for constructing the model were 9 species of the genus Campanula L. from the collection of ornamental plants of the Central Siberian Botanical Garden SB RAS

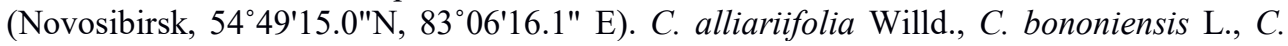
carpatica Jacq., C. lactiflora M. Bieb., C. punctata Lam., C. sarmatica Ker-Gawl., C. takesimana Nakai, C. trachelium L., and C. rapunculoides L. are herbaceous summerflowering polycarpics, cultivated as ornamental perennials. All species annually form elongated reproductive shoots with terminal multi-flowered inflorescences synflorescences. Inflorescences of Campanula are considered as belonging to a transitional group between cymose and racemose ones due to a number of morphological features: the presence of both inflorescences in the same species, multi-flowered lateral axes, and variable sequence of flowering [11]. Inflorescences are simple or compound, closed (if the main axis ends in a terminal flower) or rarely open.

Our description of inflorescence structure followed modern approaches [2 ]. Inflorescences of Campanula species represent two main types, such as a simple closed brush - botryoid, and a panicle. The reproductive shoot is usually ends with a terminal flower, below which is a zone of the 1st order axes (paraclades). These are the lateral flowers on pedicels of different lengths. The latter can branch to 2nd order axes and less frequently to higher order axes. The sequence of flowering in the inflorescence is not the same. Most often, the terminal flower opens first followed by several flowers in the lower third, and further opening is divergent (C. carpatica, C. lactiflora, C. punctata, $C$. sarmatica, $C$. takesimana, $C$. trachelium). In the inflorescences of $C$. alliariifolia, $C$. bononiensis, and C. rapunculoides - in these species they may be open - flowering begins in the lower third and follows in a divergent sequence, but the terminal flower is always ahead of 2-4 lower flowers. The terminal flowers on the elongated paraclades also open first.

Phenological observations were carried out on three shoots of each species regularly during the flowering period. The inflorescence structure was described at the end of the growing season using a sample of 10 shoots for three years. The choice of dependences for constructing the model was determined on the analysis of phenological data. All calculations related to obtaining standard statistical parameters of the model were made in the original $\mathrm{C}++$ program in Linux. All graphs and diagrams were obtained using the gnuplot graphical utility [12]. 
A detailed description of the mathematical formalism of the model, a demo version of the $\mathrm{C}++$ code that implements the model, and diagrams illustrating both the quality of the model and the flowering features of the species studied can be downloaded from https://github.com/fomined/ornamental.

\section{Results and discussion}

\subsection{Model of individual flowering}

The model of individual flowering is based on the approach proposed earlier [10]. We offer an improved version of the model, that:

- includes more dependences between the phenological parameters of flowering;

- uses nonlinear regressions instead of linear regressions to approximate dependences;

- smoothes the empirical probability distributions numerically without fitting to any distribution function.

This model takes into account:

- the probability distributions of the number of flowers on the 2 nd order axes of shoots and on the shoots on the whole;

- the probability distributions of the duration of the phases of development (bud, flower, and ovary) of flowers on the 1st and 2 nd orders axes separately;

- regression dependence of the number of flowers on the 2 nd order axes from the number of flowers on the 1st order axes;

- relative density of flowers on the 1 st and 2 nd order axes depending on the relative height in the inflorescence;

- time of the beginning of the phases of development (bud, flower, and ovary) for flowers on the 1st and 2 nd orders axes depending on their relative height;

- the duration of the phases of development for flowers on the 1 st and 2 nd order axes depending on the relative height.

To construct the dependences, lowess curves [13] were used, which allow to select the deterministic part from stochastic data accurately. The advantages of these curves stems from the use of a large set of simple regression models that approximate the data locally, and the overall curve is the result of their sewing together. . As a consequence, to describe the order of flower opening on any axis of the inflorescence, instead of several regressions fitted to different parts of the shoot, it becomes possible to use a single regression curve. All curves of the model are automatically recalculated when changing data files, so the model becomes adaptive and portable to other species. The flexibility of lowess curves provides high accuracy and reproducibility of model curves (see Table 1), although their use requires significantly greater computational costs.

Table 1. The coefficients of determination $\mathrm{R}^{2}$ of the models. The upper value corresponds to [10], and the lower - to the proposed model. The average values for observed shoots with the error $(\mathrm{M} \pm$ m) are given

\begin{tabular}{|l|c|c|}
\hline \multicolumn{1}{|c|}{ Indicator } & C. bononiensis & C. sarmatica \\
\hline Flower opening curve on 1st order & $0.81 \pm 0.07$ & $0.59 \pm 0.33$ \\
axes & $\mathbf{0 . 8 9} \pm \mathbf{0 . 0 4}$ & $\mathbf{0 . 8 5} \pm \mathbf{0 . 1 1}$ \\
\hline Flower opening curve on 2st order & 0.35 & 0.20 \\
axes & $\mathbf{0 . 6 1} \pm \mathbf{0 . 1 3}$ & $\mathbf{0 . 7 4} \pm \mathbf{0 . 1 5}$ \\
\hline Flowering curve & 0.83 & 0.77 \\
& $\mathbf{0 . 9 9 6} \pm \mathbf{0 . 0 0 1}$ & $\mathbf{0 . 9 8 7} \pm \mathbf{0 . 0 0 3}$ \\
\hline
\end{tabular}


In multi-flowered inflorescences, a multi-index grouping of flowers is used, which indicates the location of each flower on the axis of any order (Fig. 1). For convenience of data processing, the terminal flower of the main axis and flowers on 1st order axes receive continuous numbering and are further considered together as flowers of the 1 st order. The lateral flowers of paraclades are numbered by double indexes and make up a collection of flowers of the 2 nd order. For each flower in the inflorescence, on each observation date, the developmental phase is noted: bud, flower, and ovary.

Using the extracted dependences, the software implementation of the model allows to generate the dynamics of the development of simulated shoots. This makes it possible, firstly, to evaluate the quality of the model by comparing the flowering curves of observed and simulated shoots, and secondly, to simulate the flowering of shoots with an arbitrary number of flowers on axes of different orders.

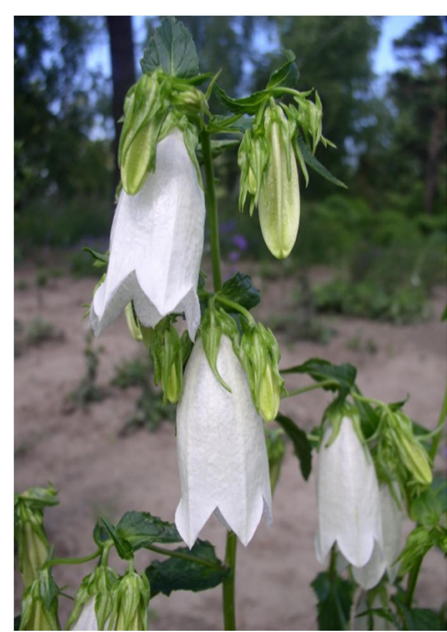

A

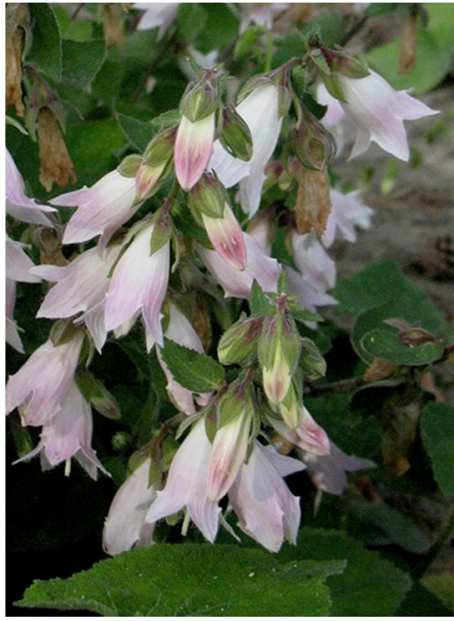

B

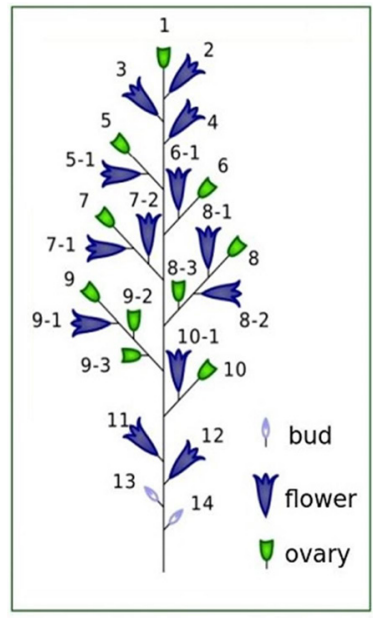

$\mathrm{C}$

Fig. 1. Inflorescences of Campanula species: A - C. punctata, B - C. alliariifolia; C - panicle scheme: 1 - terminal flower, $2 \ldots 14$ - flowers on the 1 st order axes, $5-1 \ldots 10-1$ - flowers on the 2 nd order axes.

\subsection{Flowering patterns}

Biological patterns are interpreted as stable, regularly recurring, dynamic elements of a phenomenon. Flowering patterns can be described in various ways, including computer modeling. In this case, regression curves and flowering curves are used. Analysis of the observed data (see additional materials) revealed a number of flowering features of Campanula species:

- the probability distributions of any flowering parameter (the number of flowers on the axes of different orders and thetotal number of flowers on shoots, the duration of any phase of flower development, etc.) differ from the normal distribution, in some cases significantly;

- the total number of flowers on the 2 nd order axes is positively correlated with the number of flowers on the 1 st order axes in most species;

- the beginning and duration of the developmental phases of each flower on the shoot, as well as the duration of flowering of the shoot as a whole, weakly depend on the total number of flowers. 
The dependence of the opening time of flowers on their location in the inflorescence reflect the regression curves. In the case when flowering begins in the lower third of the inflorescence, the regression curve for flowers on the 1st order axes has a C-shaped appearance, due to the delay in flowering both above and below flowers (Fig. 2, A). If flowering begins with a terminal flower, the regression curve is S-shaped. The upper loop in 'S' shows the delay of several flowers under the terminal one, and the lower loop shows the divergent order of flowering (Fig. 2, B). These regression curves are one of the most characteristic flowering patterns of Campanula species.

It was also revealed that in most species there is an increasing delay the onset of flowering of the 2 nd order axes relative to the 1st order axis (lateral flowers on the paraclade lag behind the terminal) with an increase in the distance from the point of the flowering beginning of the shoot (Fig. 2).

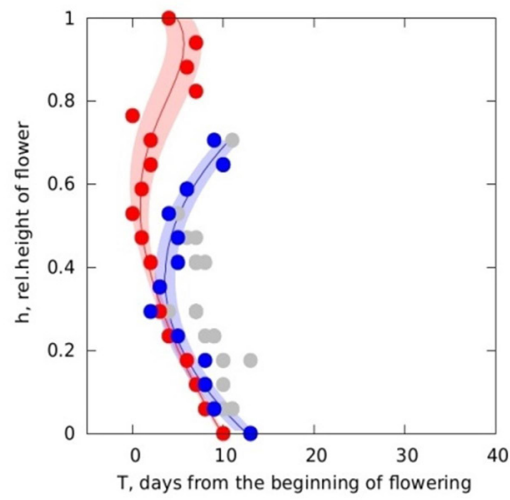

A

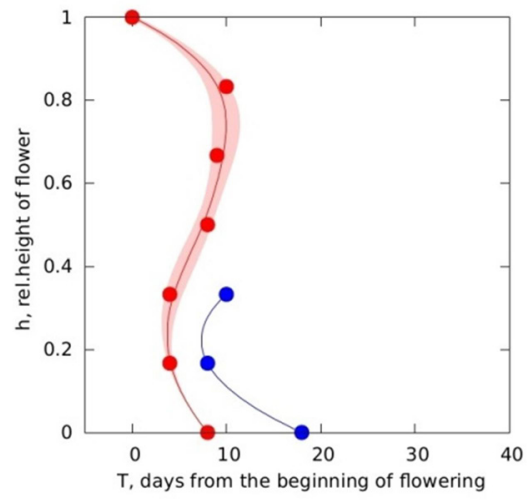

$\mathrm{B}$

Fig. 2. Regression curves of shoot flowering in Campanula species: A - C. trachelium; B - C. punctata. The regression curves of opening the flowers on the 1 st order axes depending on their height in the inflorescence are highlighted in red, and the flowers on the 2nd order axes - in blue. Dots mark the flowers in the beginning of flowering. Wide color bands indicate the standard deviation of the curves from the experimental data.

It was found that the flowering curves of Campanula species are not the same and are primarily due to the degree of development of the inflorescence (Fig. 3). In simple inflorescences that include only 1 st order flowers or also single 2 nd order flowers, the

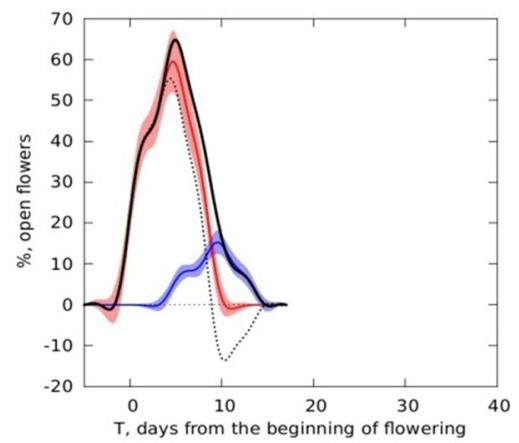

A

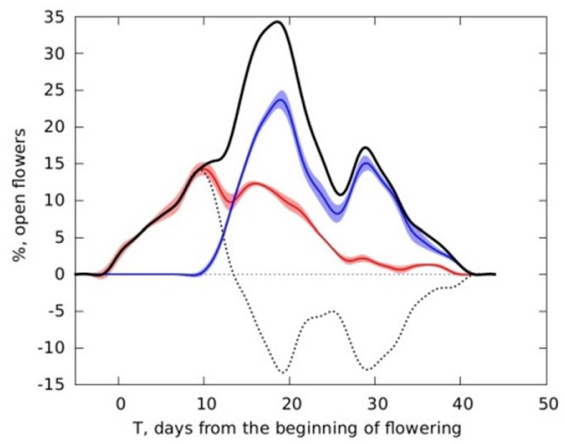

B

Fig. 3. Shoot flowering curves in Campanula species with different inflorescence structures: $\mathrm{A}-C$. punctata, simple inflorescence, unimodal type; B - C. alliariifolia, complex inflorescence, bimodal type. Red indicates the flowering curve of the 1 st order axes, blue - the 2 nd order axes, and black - 
the general flowering curve of the shoot. The dashed line shows the difference curve of the flowering transition from the 1st order axes to the 2nd order axes. Wide bands mean the standard deviation of the flowering curves.

flowering curve has a single peak, i.e. it is unimodal. In compound inflorescences, it can be bimodal - there are two flowering maxima associated with the opening of flowers on the axes of the 1 st, then 2 nd order. In the developed inflorescences, where morphogenesis processes are not completed by the beginning of flowering (C. alliariifolia, C. carpatica, $C$. rapunculoides), and therefore the flowering is long, the curve has a wavy (multimodal) character with many "bursts" (Fig. 4). The number of simultaneously opening flowers characterizes a friendly or extended type of flowering. Variations of the flowering curves for different shoots are manifested in the height of the peaks, their number, and the length of the intervals between them. The diversity of individual flowering patterns in Campanula species is largely due to the transitional type of their inflorescences.

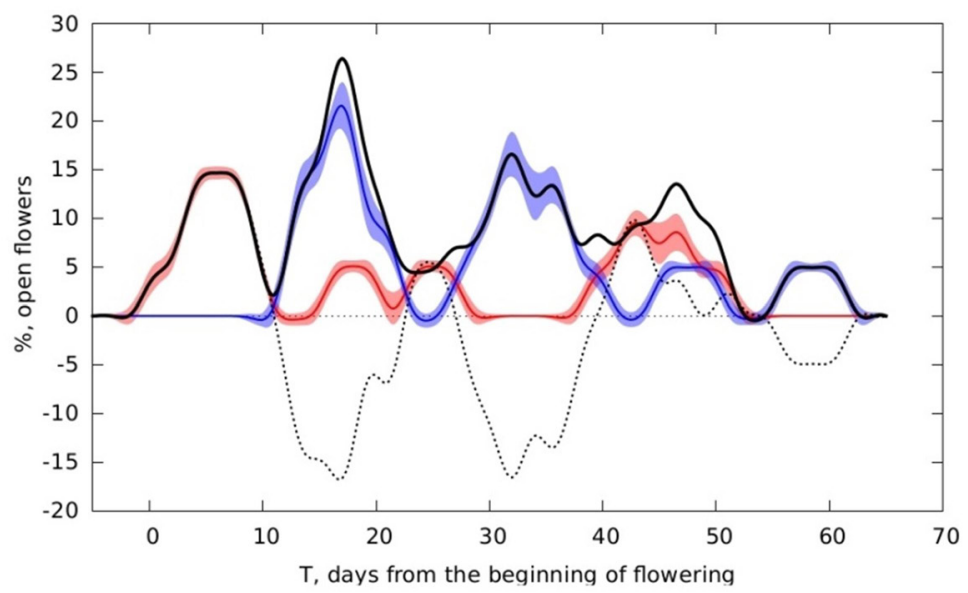

Fig. 4. Multimodal flowering curve of C. carpatica shoot. Symbols - see Fig. 3.

\section{Conclusion}

On the example of Campanula species, it was shown that computer modeling allows to process and informatively present the data of phenological observations at a modern level, revealing flowering patterns. Using the dependences of the phenological parameters of flowering at the shoot level, the model is able to predict the flowering dynamics of individuals and their groups by generating simulated shoots with an arbitrary number of flowers on the axes of different orders. Flowering patterns should be considered as a significant phenotypic trait for characterizing of herbaceous biomorphs.

The article was prepared using materials from the Bioresource scientific collection of the Central Siberian Botanical Garden SB RAS, "Collections of living plants in open and closed ground", USU 440534. This work was performed within the framework of state assignments No. 0312-2016-0003 and No. 0324-2019-0040-S-01.

\section{References}

1. A. L. Takhtajyan. Questions of evolutionary morphology of plants (L., 1954)

2. L.D. Harder, P. Prusinkiewicz, Ann. Bot. 112, 1477 (2013) 
3. P.Yu. Zhmylev, E.A. Karpuhina, A.P. Zhmyleva, Zhurnal Obshchei Biologii, 70, 262 (2009)

4. B. Rathcke, E.P. Lacey, Annu. Rev. Ecol. Evol. S., 16, 179 (1985)

5. R.B. Primack, Patterns of flowering phenology in communities, populations, individuals, and single flowers: The population structure of vegetation (Ed. J. White. Dordrecht, 1985)

6. J. Erwin. Factors affecting flowering in ornamental plants: Flower breeding and genetics (issues, challenges and opportunities for the 21 st century), (Ed. N.O. Anderson. Minnesota, 2007)

7. I.G. Serebryakov, Botanicheskii Zhurnal, 51, 923 (1966)

8. D.A. Afonnikov, M.A. Genaev, A.V. Doroshkov, E.G. Komyshev, T.A. Pshenichnikova, Russ. J. Genet., 52, 688 (2016)

9. L. Li, Q. Zhang, D. Huang, Sensors, 14, 20078 (2014)

10. E.S. Fomin, T.I. Fomina, Vavilov J. of Genetics and Breeding, 22, 845 (2018)

11. R. Carolin., Proc. Linn. Soc., 92, 7 (1967)

12. T. Williams, C. Kelley, Gnuplot 4.6: an interactive plotting program (2013)

13. W.S Cleveland., J. of the American Statistical Association, 74, 368 (1979) 